\title{
Editorial
}

\section{Multiple Criteria Decision Making Theory, Methods, and Applications in Engineering}

\author{
Ching-Ter Chang, ${ }^{1}$ Kim-Hua Tan, ${ }^{2}$ and Hao-Chun $\mathrm{Lu}^{3}$ \\ ${ }^{1}$ Graduate Institute of Business and Management, Chang Gung University, 259 Wen-Hwa 1st Road, Kwei-Shan, Tao-Yuan 333, Taiwan \\ ${ }^{2}$ Operations Management MSc Programme, Nottingham University Business School, Jubilee Campus, Nottingham NG8 1BB, UK \\ ${ }^{3}$ Department of Information Management, Fu Jen Catholic University, No. 510, ZhongZheng Road, Xinzhuang District, New Taipei \\ City 24205, Taiwan
}

Correspondence should be addressed to Ching-Ter Chang; chingter@mail.cgu.edu.tw

Received 4 June 2014; Accepted 4 June 2014; Published 21 July 2014

Copyright ( 92014 Ching-Ter Chang et al. This is an open access article distributed under the Creative Commons Attribution License, which permits unrestricted use, distribution, and reproduction in any medium, provided the original work is properly cited.

Multiple criteria decision-making (MCDM) is a subdiscipline of operations research that explicitly considers multiple criteria in decision-making environments. Be it in our daily lives or in professional engineering settings, there are typically multiple conflicting criteria that need to be evaluated in making decisions.

This special issue intends to provide the details of recent advances of MCDM and promote the applications of MCDM methods in the engineering context. This special issue also provides a forum for researchers and practitioners to review and disseminate quality research work on MCDM problems and their applications in the context of engineering and manufacturing operations and to identify critical issues for further developments.

In this special issue we have invited investigators to contribute original research articles as well as review articles on all aspects of MCDM approaches and fuzzy multiobjective programming methods. Of particular interest are papers devoted to the development of advanced MCDM methods for direct or indirect use in engineering, decision science, and manufacturing operations settings. Contributions containing computational issues, search strategies, and modeling and solution techniques to practical problems are also encouraged. In total, there were 47 submissions from research institutions in various continents, and only 23 manuscripts were accepted. Thus, the acceptance rate is less than $50 \%$. The accepted manuscripts in this special issue were of very high quality and can be classified into four main MCDM categories: (1) multiobjective mathematical programming, (2) multiattribute utility theory, (3) outranking relations approach, and (4) preference disaggregation approach.

We are extremely pleased to bring you this special issue. Our initial worries were clearly without foundation; many fine scholars produced outstanding and useful research and ideas for this issue.

\section{Acknowledgment}

All the papers submitted to this special issue were peer reviewed by two or more referees. We are very grateful to the referees for their support and service, as without them, this special issue would not have been possible.

Ching-Ter Chang Kim-Hua Tan Hao-Chun Lu 


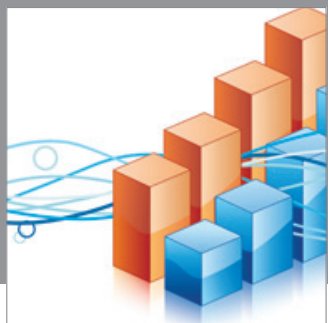

Advances in

Operations Research

mansans

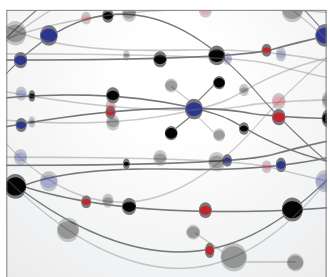

The Scientific World Journal
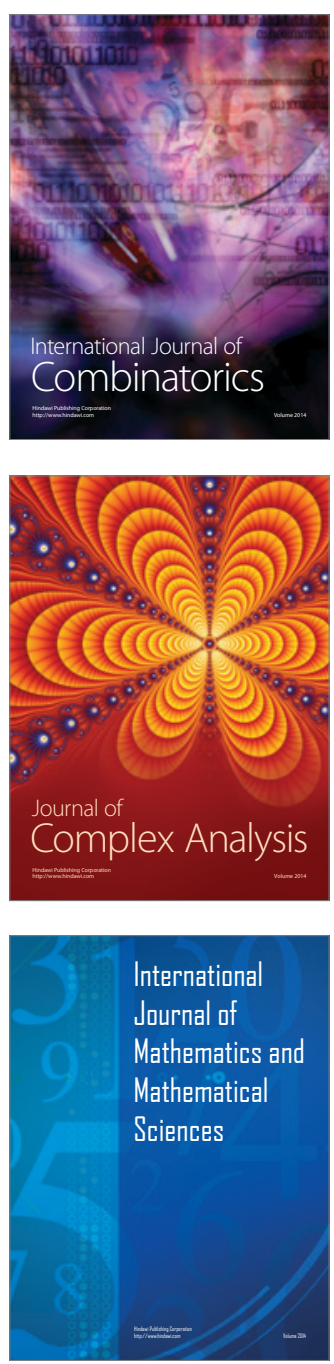
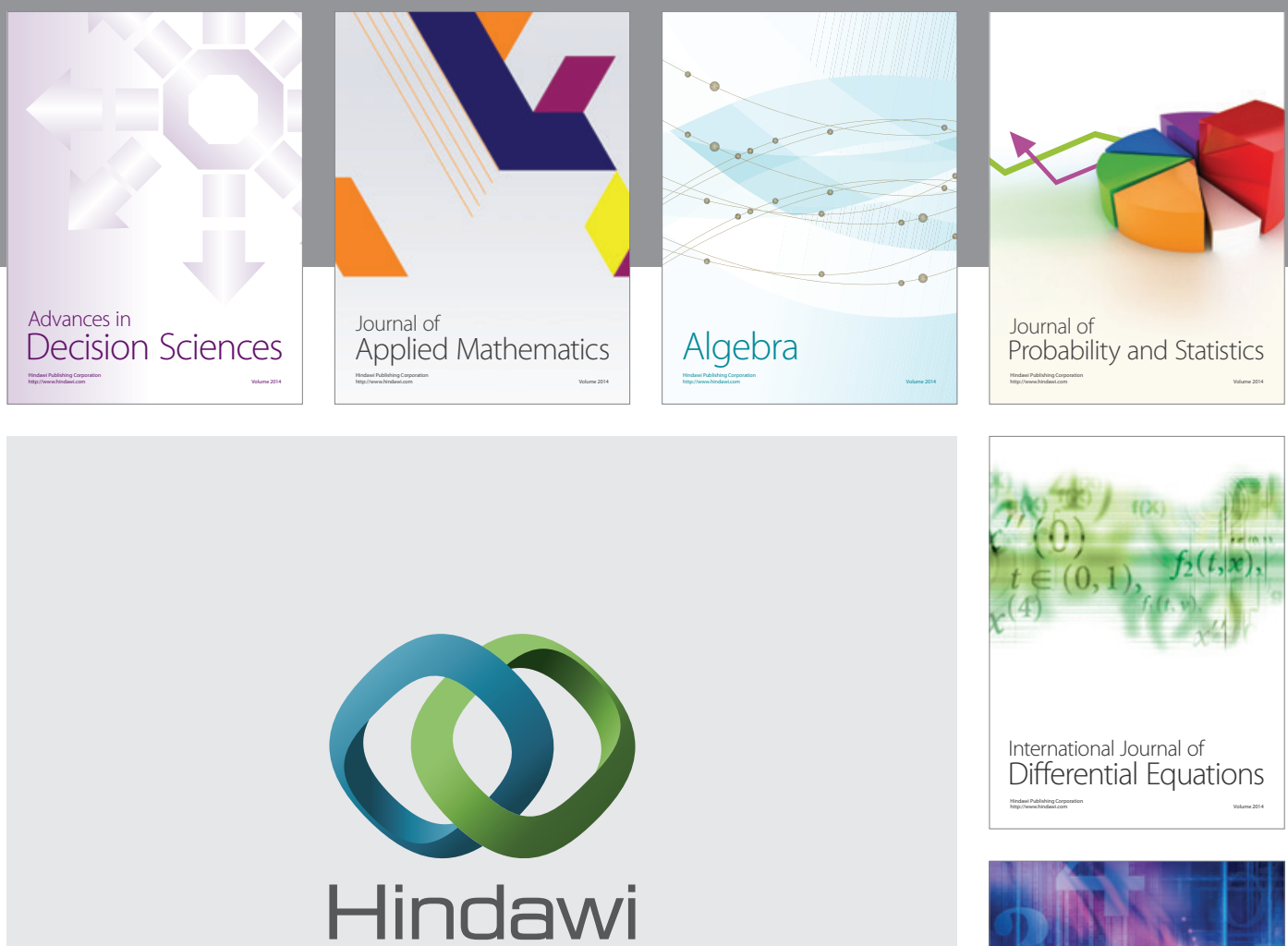

Submit your manuscripts at http://www.hindawi.com
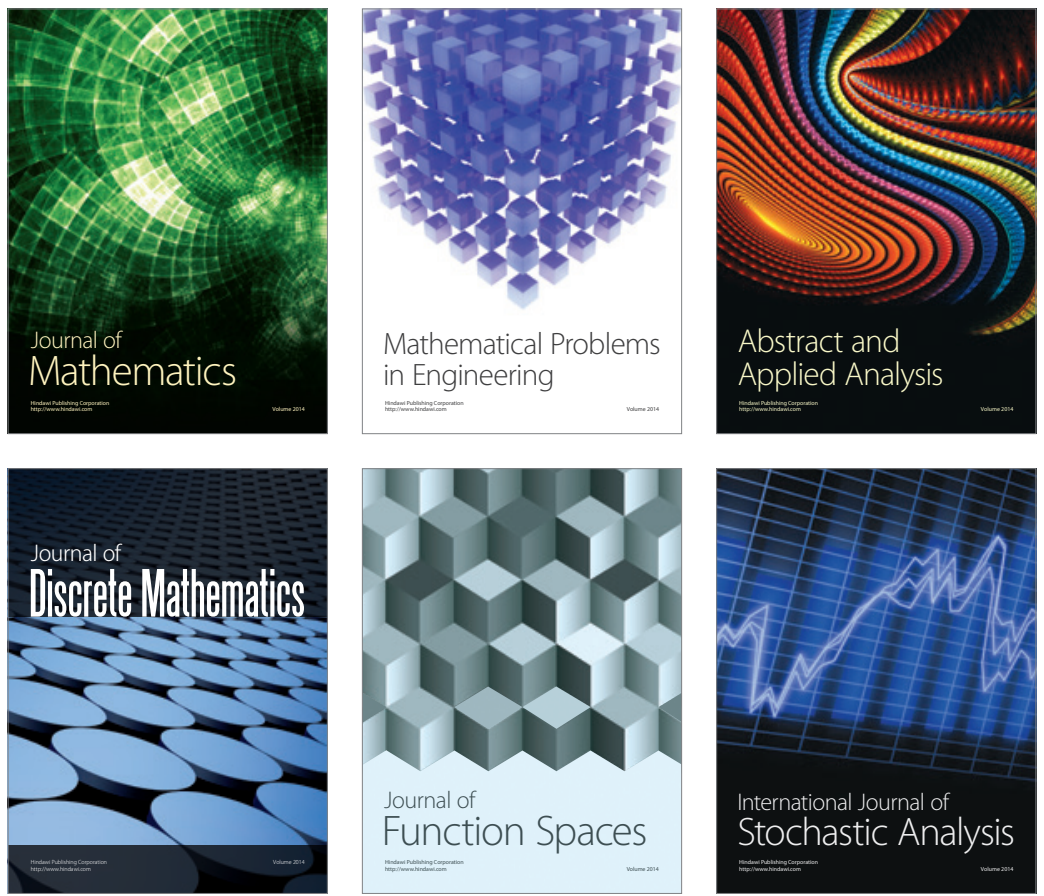

Journal of

Function Spaces

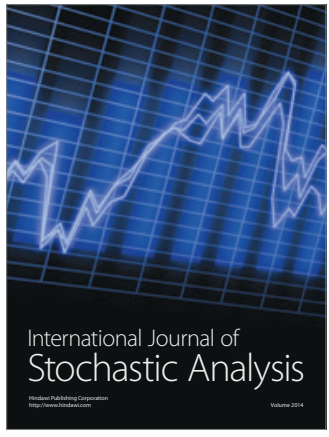

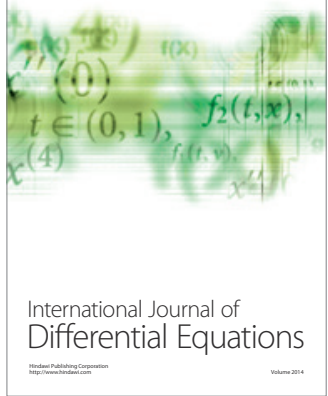
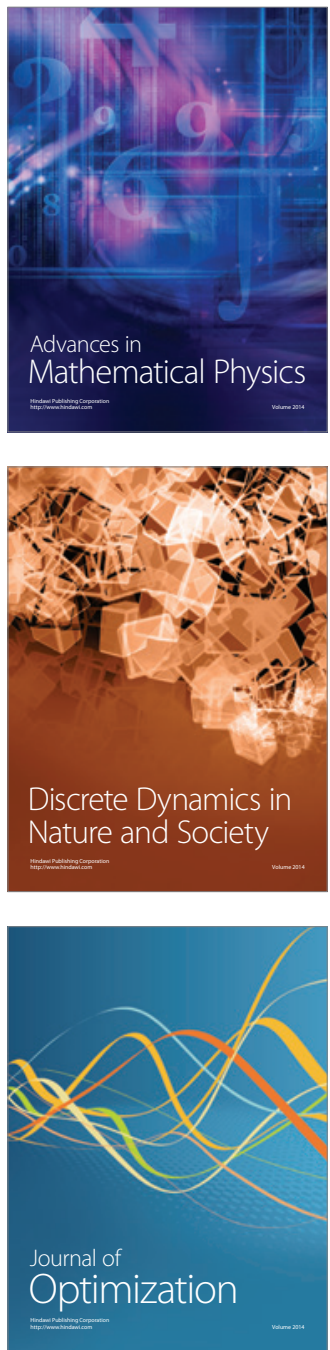